\title{
Wireless Sensor Traffic Information Collection System Based on Congestion Control Algorithm
}

\author{
https://doi.org/10.3991/ijoe.v13i11.7751 \\ Hongfeng Sun \\ Shandong Women's University, Shandong, China \\ nameshf@163.com
}

\begin{abstract}
In order to develop a fast and convenient traffic information collection system, the wireless sensor traffic information collection system based on congestion control algorithm is designed. This new system reduces the operation and maintenance costs and improves the efficiency of traffic information collection. By analyzing the wireless sensor network, the application of wireless sensor network in intelligent traffic information collection system is discussed. In addition, the design scheme of traffic information collection system is designed. After analyzing the size of the network in the scheme, the congestion problem in the scheme is discussed finally. The simulation results show that the node communication radius is $50 \mathrm{~m}$ when the simulation scene is located in the monitoring area, while the MAC protocol is $802.11 \mathrm{DCF}$ and the initial energy of all nodes is $1 \mathrm{~J}$. Based on the above finding, it is concluded that the congestion control algorithm has better throughput, network delay and network energy consumption, which is suitable for the wireless sensor traffic information collection system.
\end{abstract}

Keywords-Traffic information collection; wireless sensor network; congestion control; shunt regulation

\section{Introduction}

With the rapid development of urban modernization, people's demand is constantly rising. The convenient, comfortable and safe traffic environment has been pursued by adults. However, the increasingly serious traffic problem has become an obstacle to the rapid development of the city. In recent years, the volume of traffic in major cities has been on the rise. Taking Shanghai city as an example, the daily traffic flow of urban roads has increased from 1 million 500 thousand to 3 million passengers. Especially during the Expo, the traffic congestion caused by the traffic congestion in the urban central area is becoming more and more serious. Traffic safety, traffic congestion, pollution and other issues not only decrease the efficiency of traffic of the city, but also cause huge economic losses and serious environmental pollution and other social problems. These factors impact on people's quality of life significantly. The increasingly serious traffic problems in the world's major cities have become a social problem that cannot be ignored. It seriously restricts the pace of economic develop- 
ment and the process of modernization and civilization. The primary problem of urban development is how to take effective measures to solve the traffic problems. According to these social demands, traffic information collection and intelligent transportation system emerge as the times require. It is the basis for traffic state judgment and prediction, traffic flow control and emergency response [1].

\section{Method}

\subsection{Overview of WSN congestion control}

Wireless sensor networks (WSN) are widely used in many fields because of their low cost, easy local management and easy expansion [2]. Work modes can be divided into on-demand query mode, periodic reporting model, and event driven mode. In ondemand query mode, wireless sensor networks are generally deployed around the monitoring area and are normally asleep. When the user needs to know the situation of the monitored environment, it will be activated, collected, processed and transmitted, and finally transmit the data that meets the requirements to the user. In the periodic reporting mode, the wireless sensor network will periodically monitor the relevant information of the target object, and then transmit the monitoring results to the user through the scheduled time cycle. In unexpected event driven mode, because the probability of unexpected events is very low, the traffic flow is normal in general. But in the event of unexpected events, the network traffic changes greatly, and the sensor nodes will report the relevant situation quickly and initiatively, which is helpful for the accurate judgment of unexpected events. However, in the above three modes, there will be some congestion. At this point, a solution to the problem is how to quickly and effectively alleviate and avoid congestion. Congestion control in wireless sensor networks has been on the rise in recent years. Mainly because of the different applications in the actual environment, it causes a variety of network congestion problems. Therefore, the solution of congestion is urgent. Congestion control technology is to avoid congestion or to make rapid and effective control of congestion through certain technical means.

\subsection{Analysis of WSN congestion control algorithm}

In view of the characteristics of wireless sensor networks, a variety of congestion control algorithms have been proposed by scholars both at home and abroad. ESRT [3] protocol is the first-rate control based congestion control protocol. It combines reliability and energy consumption into consideration, and achieves the purpose of mitigating congestion through proper adjustment of speed. At the same time, it improves reliability and saves energy. But it requires the gateway node to have the communication capability to cover the entire network. It also uses simple packet count to measure reliability, and does not distinguish the contribution of data sources to the fidelity of information. All nodes adopt a unified adjustment scheme. COAD [4] can adjust the congestion caused by local nodes, and use hop by hop control to 
reduce energy consumption. But because of the feedback operation of gateway and multiple nodes, especially in large-scale network, the speed of closed loop control is relatively slow. ARC [5] protocol is a multiway split protocol based on traffic scheduling. It creates multiple paths to increase the accuracy of the transmitted packets, avoids conflicts and retransmissions, and greatly saves the energy of nodes. CAR [6] protocol is similar to the ARC protocol, and it is suitable for many different priority data stream networks. But it lacks support for high mobile data sources, and it costs a lot to build high priority data routing. BGR [7] protocol is also a control protocol combined with multi-path routing. Compared with other protocols, its implementation mechanism is relatively simple, but the drawback is that it lacks the comprehensive consideration of the current situation of the neighbor nodes, resulting in the expansion of the surrounding congestion area. CONCERT [8] protocol uses the appropriate data aggregation method to reduce data traffic in the network to mitigate congestion, so as to achieve the purpose of prolonging the network cycle [9]. In recent years, some scholars have tried to propose an active queue management mechanism for wireless sensor networks based on system control and optimization [10]. Active queue management technology predicts the possible congestion through the current state of the network, and notifies the source in advance by dropping or marking the packet to reduce the sending rate, thus achieving the purpose of congestion avoidance.

\subsection{Congestion control algorithm for wireless sensor networks based on TOPSIS}

Based on the above analysis, in order to ensure the real-time and reliability of traffic data acquisition, a high-performance congestion control algorithm is proposed in this paper. In view of the fact that single congestion detection cannot predict the network load accurately, the algorithm defines congestion prediction state index according to queue occupancy and congestion duration. And through the judgment of the congestion status index, the congestion trend is predicted accurately. In order to avoid the network failure caused by the failure of the optimal path, the algorithm constructs the selection model based on the theory of ding. A temporary optimal path is established around the congestion nodes to adjust the congestion level and analyze the congestion level. The residual energy, the distance from the original path, and the channel access rate are chosen as the basis for the next hop node. Experimental results show that the proposed algorithm can detect congestion effectively and accurately, reduce latency and network energy consumption. It guarantees the fidelity of the data and further improves the performance of the traffic information collection system.

TOPSIS theoretical model. The full name of TOPSIS [11] is "ranking method approaching to ideal value ". As the name suggests, it is a comprehensive decisionmaking method [12]. Through the analysis of a number of attributes, each program is sorted, and finally a scheme approaching to the ideal value is obtained. The basic ideas are as follows: first, the raw data is unified by normalization. Secondly, the attribute weighting matrix is determined to form a judgment matrix [13]. Then, the optimal scheme and the worst scheme are determined, and the degree of closeness between the optimal scheme and the worst scheme is obtained by calculating the dis- 
tance between the optimal scheme and the worst scheme. The above data is taken as an index of the research object, the general steps of TOPSIS analysis method are as follows:

$$
V=\left|\begin{array}{cccc}
V_{11} & V_{12} & V_{13} & V_{14} \\
V_{21} & V_{22} & V_{23} & V_{24} \\
\cdots & \cdots & \cdots & \cdots \\
V_{\mathrm{m} 1} & V_{m 2} & V_{m 3} & V_{m 4}
\end{array}\right|
$$

2) The normalized value of the node property in formula (1) is:

$$
v_{i j}=x_{i j} / \sqrt{\sum_{i=1}^{m} x_{i j}^{2}}, i=1,2,3, \ldots m ; j=1,2,3,4
$$

Congestion control mechanism based on TOPSIS. Road traffic information collection system has the following characteristics: A large number of sensors are used on both sides of the road to monitor traffic flow data in real time. The nodes need to transmit the monitored road traffic data to the sink node quickly and accurately with multi hop or multiple to one transmission. Generally, sensor nodes and sink nodes do not change positions after deployment. In the event of unexpected events, a large amount of data will be generated in the sensor network. Wireless sensor networks for traffic information collection are different from those of general monitoring networks. In traffic information collection, some data is very important, and it is not allowed to reduce speed. It needs to be transmitted to monitoring center immediately. At the same time, the sensor covers a wide range of data streams and has a large number of redundant nodes. Because of these characteristics, the general congestion control is difficult to meet its needs. However, the traffic scheduling control method can schedule the data flow into the congestion region without decreasing the speed, so as to ensure the original transmission line smooth through the detour, relieve congestion diversion. Fast and accurate congestion detection is the premise and foundation of congestion control.

\section{$3 \quad$ Algorithm simulations}

\subsection{Introduction to simulation tools}

In the research of wireless sensor network technology, simulation is an effective and economic research method. The NS2 used in this paper is a free software simulation platform for network technology, open source code. Through the use of NS2, the computer staff can develop network technology simply and quickly. The modules contained in NS2 almost involve all aspects of network technology. Therefore, NS2 has become a widely used network simulation software in academia. In addition, NS2, 
as an auxiliary teaching tool, has been widely used in the teaching of network technology.

\subsection{Simulation environment settings}

In order to verify the accuracy of the model, the simulation results of the algorithm are compared and analyzed. NS2 simulation software is used to construct multi hop wireless sensor networks. Based on the above considerations, the simulation scene is located in the monitoring area, and the node communication radius is $50 \mathrm{~m}$. The MAC protocol is $802.11 \mathrm{DCF}$ and the initial energy of all nodes is $1 \mathrm{~J}$. In addition, the cache size is 80 packets, the congestion threshold is 0.8 , and the packet size is 512 bytes.

\section{$4 \quad$ Result and discussion}

The real time and reliability of data transmission is an important performance evaluation index of WSN, and throughput reflects the degree of reliability of the network. By comparing the network throughput of CODA, CAR and HPCC, it can be concluded that the throughput of the three protocols is basically consistent within 0-30 seconds. The reason is that the source rate is low and the network capacity can hold the data traffic at this time. As a result, congestion can only be avoided. After 30 seconds, the source node delivery rate and the network data traffic flow increase, causing network congestion. At this point, CAR and HPCC quickly adjust the resource supply and establish a new path. After sharing the traffic for the original path, the throughput is improved. However, CODA can alleviate congestion by adjusting the upstream node and source node rate, so that the throughput does not increase with the increase of the source data flow. Since the channel access rate is chosen as the next hop node, the throughput of HPCC is higher than CAR. At the same time, the best next hop node is selected through the TOPSIS multi-attribute decision model. Therefore, the new path is better than the path built by CAR, so throughput performance has been improved. Figure 1 shows the average end-to-end delay contrast of CODA, CAR, and HPCC. In the initial stage, the packets in the buffer space are accumulating constantly, which leads to the increase of queue waiting time, so the delay of the algorithm increases rapidly. After the congestion, CAR and HPCC create a new path to share the traffic for the original path and reduce the queue length of the node cache, thus reducing the queuing time of the packets in the queue of nodes. However, CODA inhibits upstream node forwarding rates. Although the queue length of congestion nodes is shortened, the delay of packet transmission in upstream nodes is increased. Therefore, the average end-to-end delay becomes larger and the delay is higher than that of CAR and HPCC. After 50 seconds, the network delay is not rising, this is because the source traffic gradually become stable. After the new path is established, the traffic flow is shared for the original path, and the network load state tends to be stable. The HPCC delay in the figure is about $20 \%$ lower than that of CAR. Because the congestion prediction state index is adopted to judge the network state quickly and accurately, the performance of HPCC delay is better than CAR. The main feature of 
WSN is that it takes full account of the limited energy of nodes. The main feature of WSN is that it takes full account of the limited energy of nodes. Under the premise of ensuring the reliability of data transmission, users should minimize the energy consumption on the communication path, which will improve the energy efficiency and maximize the network lifetime.

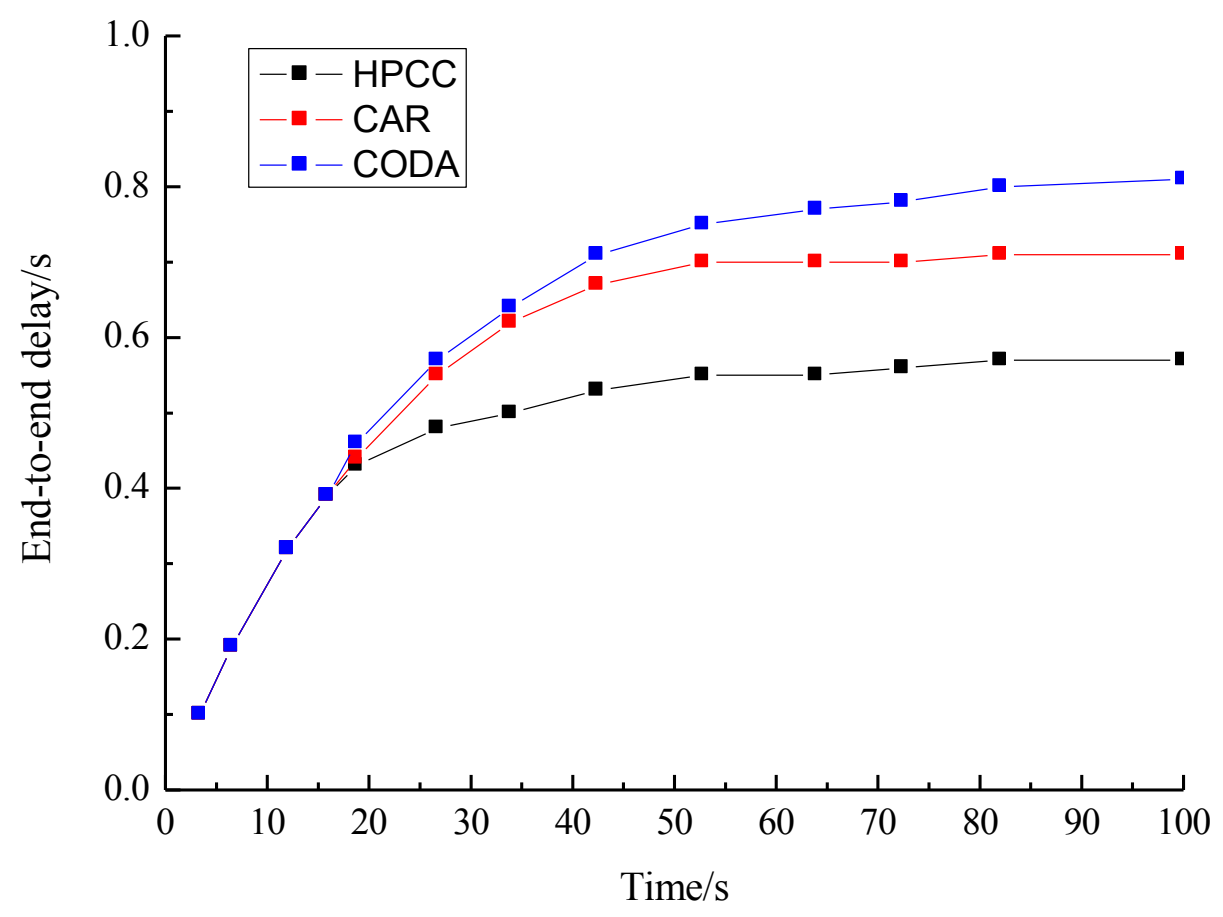

Fig. 1. Comparison of average end-to-end delay of CODA, CAR and HPCC

As shown in figure 2, the energy consumption rate of HPCC is slower than that of CODA and CAR. Because CODA involves the feedback interaction between gateways and multiple nodes, the energy consumption is greater. CAR creates a diversion path that consumes a lot of energy. The HPCC uses the TOPSIS model to select fast triage, while periodically scanning cache queues are also being performed with relatively small energy consumption. Network resources can be used effectively and show better energy saving. 


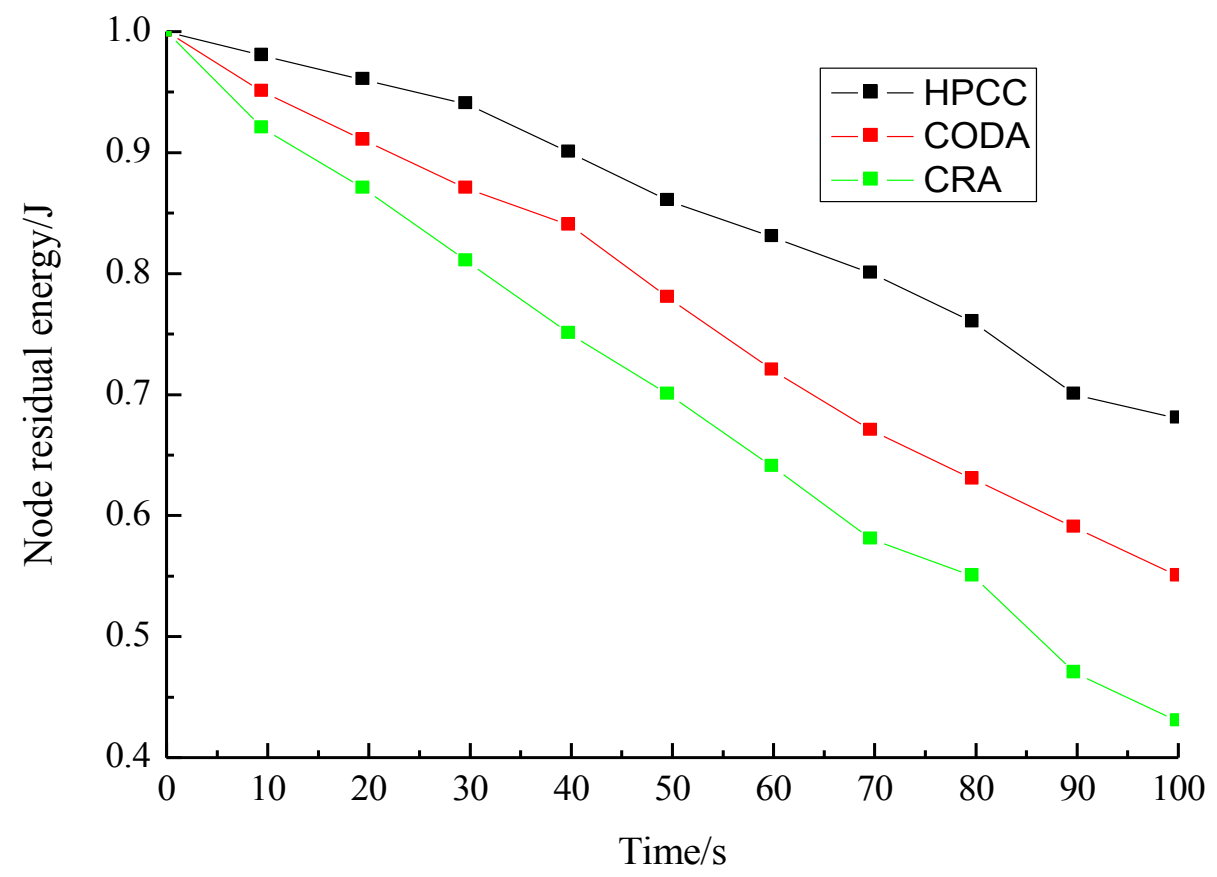

Fig. 2. Comparison of average end-to-end delay of CODA, CAR and HPCC

\section{Conclusion}

The wireless sensor networks for traffic information collection is studied, which aims to improve congestion in wireless sensor networks. In order to improve the network performance, the network model is established and the characteristics of the algorithm are analyzed. At present, most congestion control algorithms change the transmission speed of nodes to control congestion, but there is little research on multipath shunting due to the constraints of application environment and the unpredictable nature of protocols. The main work of this paper includes:

1. Through the analysis of the requirements of the information collection system, the network architecture and networking scheme of the wireless sensor network for traffic information collection are designed, and some key problems of the wireless sensor network are analyzed.

2. Aiming at the congestion problem of wireless sensor networks for traffic information collection, a high performance congestion control algorithm for traffic information collection is proposed on the basis of traffic scheduling.

3. The simulation verification of congestion control algorithm is implemented by using network simulation software. Compared with the existing congestion control algorithms, it shows that it cannot only solve the congestion problem better, but also make full use of redundant resources. Therefore, this new congestion control algorithm has better throughput, network delay and network energy consumption. 


\section{References}

[1] Gholipour, M., Haghighat, A. T., \& Meybodi, M. R. (2015). Hop-by-hop traffic-aware routing to congestion control in wireless sensor networks. Eurasip Journal on Wireless Communications \& Networking, 2015(1), 1-13. Https://doi.org/10.1186/s13638-015-0241$\underline{5}$.

[2] Prabha, R., \& Gouda, P. K. (2015). Mtadf : multi hop traffic aware data for warding for congestion control in wireless sensor networks. , 7(1), 107-118. Https://doi.org/10.5121/ijwmn.2015.7107.

[3] Manshahia, M. S., Dave, M., \& Singh, S. B. (2015). Congestion control in wireless sensor networks based on bioluminescent firefly behavior. Wireless Sensor Network, 07(12), 149156. Https://doi.org/10.4236/wsn.2015.712013.

[4] Raman, C. J., \& James, V. (2015). Fuzzy based congestion control for backpressure routing algorithm in wireless sensor networks. Research Journal of Applied Sciences Engineering \& Technology, 11(11), 1179-1189. Https://doi.org/10.19026/rjaset.11.2224.

[5] Wei, K., Guo, S., Li, X., Zeng, D., \& Xu, K. (2016). Congestion control in social-based sensor networks: a social network perspective. Peer-to-Peer Networking and Applications, 9(4), 681-691. Https://doi.org/10.1007/s12083-015-0352-0.

[6] Uthra Rajan, A., Kasmir Raja, S. V., Jeyasekar, A., \& Lattanze, A. J. (2015). Energyefficient predictive congestion control for wireless sensor networks. Wireless Sensor Systems Iet, 5(3), 115-123. Https://doi.org/10.1049/iet-wss.2013.0101.

[7] Venkataramanan, C., \& Girirajkumar, S. M. (2015). Rermac congestion control protocol for wireless sensor networks. Indian Journal of Science \& Technology, 8(35), 231-237. Https://doi.org/10.17485/ijst/2015/v8i35/70201.

[8] Park, J. H., Kim, J. H., \& Lee, S. K. (2015). A study on the enhanced congestion control mechanism for multimedia traffic in sensor networks. International Journal of Multimedia \& Ubiquitous Engineering, 10(8), 391-400. Https://doi.org/10.14257/ijmue.2015.10.8.38.

[9] Chen, W., Guan, Q., Jiang, S., Guan, Q., \& Huang, T. (2016). Joint qos provisioning and congestion control for multi-hop wireless networks. Eurasip Journal on Wireless Communications \& Networking, 2016(1), 19. Https://doi.org/10.1186/s13638-016-0519-2.

[10] Mayandi, M., \& Pillai, K. V. (2016). Probabilistic qos aware congestion control in wireless multimedia sensor networks. Circuits \& Systems, 07(9), 2081-2094. Https://doi.org/10.4236/cs.2016.79181.

[11] Chen, W., Niu, Y., \& Zou, Y. (2017). Congestion control and energy-balanced scheme based on the hierarchy for wsns. Iet Wireless Sensor Systems, 7(1), 1-8. Https://doi.org/10.1049/iet-wss.2015.0097.

[12] Duan, S., Shah-Mansouri, V., Wang, Z., \& Wong, V. W. S. (2016). D-acb: adaptive congestion control algorithm for bursty $\mathrm{m} 2 \mathrm{~m}$ traffic in lte networks. IEEE Transactions on Vehicular Technology, 65(12), 9847-9861. Https://doi.org/10.1109/icc.2015.7249249.

[13] Mandloi, M., \& Bhatia, V. (2015). Congestion control based ant colony optimization algorithm for large mimo detection. Expert Systems with Applications, 42(7), 3662-3669. Https://doi.org/10.1016/j.eswa.2014.12.035.

\section{$7 \quad$ Author}

Hongfeng Sun is with Shandong Women's University, Shandong, China.

Article submitted 24 September 2017. Published as resubmitted by the author 31 October 2017. 